\title{
An Ordering Policy for Deteriorating and Weibull Ameliorating items with Time-Varying Demand and Partial Backlogging.
}

\author{
Biswaranjan Mandal ${ }^{1}$
}

\begin{abstract}
Deterioration means decay, evaporation, and obsolescence, loss of quality or marginal value of a commodity. Obviously, deterioration decreases the usefulness of the good from its original condition. Again we know the longer the goods which are kept in inventory, the higher the deteriorating cost. Pertinently there are few research papers dealing with the inventory model under the environment of Amelioration of items. The present paper deals with an inventory model for both ameliorating and deteriorating items. The amelioration rate follows the Weibull distribution and the time-varying demand rate is assumed as over a fixed time horizon and shortages which is partially backlogged. Finally the model is illustrated with the help of a numerical example, some particular cases are derived and the sensitivity analysis of the optimal solution towards the changes in the values of different parameters is also studied.
\end{abstract}

Subject classification: AMS Classification No. 90B05, 97M40.

Key Words: Inventory, Deteriorating, Ameliorating, Weibull distribution, Timevarying demand, Shortages, and Partial backlogging.

\footnotetext{
1 Associate Professor of Mathematics, Acharya Jagadish Chandra Bose College Kolkata, West Bengal, India.
} 


\section{Introduction}

Many researchers have done their extensive research works on inventory model assuming the importance of deteriorating items. But due to less influential effect of demand, the environment of amelioration is increasing day by day. Amelioration is The natural phenomenon of ameliorating environment is observing in much life stock models which operates to make better or increase goods in quantity or amount in an inventory. A few researchers have focused on ameliorating system. The vast majority of the traditional stock models depend on the rule that the estimation of stock stays consistent after some time. This is an uncommon sort of stock model where scientists have concentrated on for both ameliorating and deteriorating things, where items ameliorate when stay at breeding yard and deteriorates when in the distribution systems. Hwang [1] developed an inventory model for ameliorating items only things independently. Mallick et al. [3] has considered a creation inventory model for both ameliorating and deteriorating items. The fast growing animals like broiler, ducks, pigs etc. in the poultry farm, highbred fishes in ponds which are known as ameliorating items. These items are quite different from the deteriorating items and so researchers make their efforts to furnish comprehensive study in different types of inventory models. In this context Moon et al[4], Law et al [5], L-Q ji [6], Valliathal et al [7], Barik S. et al [8 ], Tadj [9] are few noteworthy. Chou et al. [10] proposed an analytical solution approach is derived with different shape parameters. Mondal et al. [11] incorporated an inventory policy the effect of amelioration items with price-dependent demand. Here deterioration rate is assumed constant; two parameter Weibull distribution following ameliorating items. The assumption of a constant demand rate may not be always appropriate for many inventory items. For example milk, vegetables etc., the age of inventory has a negative impact on demand due to loss of consumer confidence on the quality of such products. On the other hand, demand of some consumer goods which are newly introduced in the market may increase with time if the customer is convinced for their quality and suitability. Thus the demand rate need not be constant always. It may be increasing or decreasing over time. In the last two decades, many researchers like Donaldson [12], Silver [13], Goswami et al [14], Buchanan [15], Datta and Pal [16], Biswaranjan Mandal [17] etc. are names to only a few to develop time varying demand models. In this paper we consider the inventory replenishment problem with time-varying demand over a fixed time horizon.

In fact an inventory policy that allows for shortage is always less expensive to operate than a policy without shortage. Many researchers like Goyal et al [18], Benkheronf [19] assumed that shortages are completely backlogged. In practice, some customers would like to wait for backlogging during that shortage period, but other would not. Consequently, the opportunity cost due to lost sales should be considered in the modelling. Wee[20], Biswaranjan Mandal [21] etc assumed that the backlogging rate was a fixed fraction of demand rate during this period. However, in some inventory system, for some fashionable commodities, the length of waiting time for next replenishment become main factor for determining whether 
the backlogging will be accepted or not. The longer the waiting time is, the smaller the backlogging rate would be. Therefore backlogging rate is variable and is dependent on the waiting time for the next replenishment.

For these sort of situations, efforts have been made to develop a realistic inventory model in presence of both ameliorating and deteriorating items. Deterioration rate is constant and amelioration rate is followed by Weibull distributed. The demand rate is considered as a time-varying demand over a fixed time horizon and shortages which is partially backlogged. Finally the model is illustrated with the help of a numerical example, some particular cases are derived and the sensitivity analysis of the optimal solution towards the changes in the values of different parameters is also performed.

\section{Notations and Assumptions}

The present inventory model is developed under the following notations and assumptions:

\subsection{Notations}

(i) $\quad \mathrm{I}(\mathrm{t})$ : On hand inventory at time $\mathrm{t}$.

(ii) $\mathrm{R}(\mathrm{t})$ : Demand rate.

(iii) $\mathrm{Q}$ : On-hand inventory.

(iv) $\theta$ : The constant deterioration rate where $0 \leq \theta<1$

(v) $\quad \mathrm{A}(\mathrm{t})$ : The ameliorating rate at time $\mathrm{t}$.

(vi) $\mathrm{T}$ : The fixed length of each production cycle.

(vii) $A_{0}$ : The ordering cost per order during the cycle period.

(viii) $p_{c}$ : The purchasing cost per unit item.

(ix) $h_{c}$ : The holding cost per unit item.

(x) $\quad d_{c}$ : The deterioration cost per unit item.

(xi) $a_{c}$ : The cost of amelioration per unit item.

(xii) $c_{s}$ : The shortage cost per unit item.

(xiii) $o_{c}$ : The opportunity cost per unit item.

(xiv) TC: Average total cost per unit time. 


\subsection{Assumptions}

(i) Lead time is zero.

(ii) Replenishment rate is infinite but size is finite.

(iii)The time horizon is finite.

(iv) There is no repair of deteriorated items occurring during the cycle.

(v) Amelioration and deterioration occur when the item is effectively in stock.

(vi) $\mathrm{A}(\mathrm{t})$ is the amelioration rate following Weibull distributed $A(t)=\alpha \beta t^{\beta-1}, 0 \leq \alpha<<1, \beta \geq 1$, where $\alpha$ is the shape parameter and $\beta$ is the scale parameter.

(vii) The time-varying demand rate is given by $\mathrm{R}(\mathrm{t})=\lambda_{0} t^{-\beta_{1}}, \lambda_{0}>0,0 \leq \beta_{1}<1$. (viii)Shortages are allowed and partially backlogged.

Here the proportion of customers who would like to accept backlogging at time $t$ is decreasing with the waiting time (T-t) waiting for the next replenishment. To take care of this situation, we have defined the backlogging rate to $\frac{1}{1+\delta(T-t)}$, when inventory is negative. The backlogging parameter $\delta$ is a positive constant.

\section{Model Formulation and Solutions}

The aim of this model is to optimize the total cost incurred and to determine the optimal ordering level. During the period $\left[0, t_{1}\right]$, the stock will be diminished due to the effect of amelioration, deterioration and demand and ultimately falls to zero at $\mathrm{t}=t_{1}$. The shortages occur during time period $\left[t_{1}, \mathrm{~T}\right]$ which are partially backlogged. The differential equations which the on-hand inventory $\mathrm{I}(\mathrm{t})$ governed by the following:

$$
\begin{aligned}
& \frac{d I(t)}{d t}+\left(\theta-\alpha \beta t^{\beta-1}\right) I(t)=-\lambda_{0} t^{-\beta_{1}}, 0 \leq t \leq t_{1} \\
& \text { And } \quad \frac{d I(t)}{d t}=-\frac{\lambda_{0} t^{-\beta_{1}}}{1+\delta(T-t)}, t_{1} \leq t \leq T
\end{aligned}
$$

The initial condition is $I(0)=Q$ and $I\left(t_{1}\right)=0$

The solutions of the equations (1) and (2) using (3) and neglecting second and higher power terms of $\theta(<1)$ and $\alpha(<1)$ are given by the following 


$$
\mathrm{I}(\mathrm{t})=Q\left(1-\theta t+\alpha t^{\beta}\right)-\frac{\lambda_{0}}{1-\beta_{1}}\left(t^{1-\beta_{1}}-\frac{\theta}{2-\beta_{1}} t^{2-\beta_{1}}+\frac{\alpha \beta}{1-\beta_{1}+\beta} t^{1-\beta_{1}+\beta}\right), 0 \leq t \leq t_{1}
$$

And $\mathrm{I}(\mathrm{t})=\lambda_{0}\left[\frac{1-\delta T}{1-\beta_{1}}\left(t_{1}^{1-\beta_{1}}-t^{1-\beta_{1}}\right)+\frac{\delta}{2-\beta_{1}}\left(t_{1}^{2-\beta_{1}}-t^{2-\beta_{1}}\right)\right], t_{1} \leq t \leq T$

Since $I\left(t_{1}\right)=0$, the expression of on-hand inventory from the equation (4) is the following after neglecting second and higher power terms of $\theta(<1)$ and $\alpha(<1)$

$$
\mathrm{Q}=\lambda_{0}\left(\frac{1}{1-\beta_{1}} t_{1}^{1-\beta_{1}}+\frac{\theta}{2-\beta_{1}} t_{1}^{2-\beta_{1}}-\frac{\alpha}{1-\beta_{1}+\beta} t_{1}^{1-\beta_{1}+\beta}\right)
$$

The total inventory holding during the time interval $\left[0, t_{1}\right]$ is given by

$$
\begin{gathered}
I_{T}=\int_{0}^{t_{1}} I(t) d t \\
=\int_{0}^{t_{1}}\left\{Q\left(1-\theta t+\alpha t^{\beta}\right)-\frac{\lambda_{0}}{1-\beta_{1}}\left(t^{1-\beta_{1}}-\frac{\theta}{2-\beta_{1}} t^{2-\beta_{1}}+\frac{\alpha \beta}{1-\beta_{1}+\beta} t^{1-\beta_{1}+\beta}\right)\right\} d t
\end{gathered}
$$

Putting the value of $\mathrm{Q}$ from (6) and integrating, then neglecting second and higher power terms of $\theta(<1)$ and $\alpha(<1)$, we get

$$
I_{T}=\lambda_{0}\left\{\frac{1}{2-\beta_{1}} t_{1}^{2-\beta_{1}}+\frac{\theta}{2\left(3-\beta_{1}\right)} t_{1}^{3-\beta_{1}}-\frac{\alpha \beta}{(1+\beta)\left(2-\beta_{1}+\beta\right)} t_{1}^{2-\beta_{1}+\beta}\right\}
$$

The total number of deteriorated units during the inventory cycle is given by

$$
\begin{gathered}
D_{T}=\theta \int_{0}^{t_{1}} I(t) d t=\theta I_{T} \\
=\theta \lambda_{0}\left\{\frac{1}{2-\beta_{1}} t_{1}^{2-\beta_{1}}+\frac{\theta}{2\left(3-\beta_{1}\right)} t_{1}^{3-\beta_{1}}-\frac{\alpha \beta}{(1+\beta)\left(2-\beta_{1}+\beta\right)} t_{1}^{2-\beta_{1}+\beta}\right\}
\end{gathered}
$$

The total number of ameliorating units during the inventory cycle is given by

$$
A_{T}=\int_{0}^{t_{1}} A(t) I(t) d t=\int_{0}^{t_{1}} \alpha \beta t^{\beta-1} I(t) d t=\frac{\lambda_{0} \alpha}{1-\beta_{1}+\beta} t_{1}^{1-\beta_{1}+\beta}
$$

The total number of shortages during the period $\left[t_{1}, \mathrm{~T}\right]$ is given by

$$
S_{T}=\int_{t_{1}}^{T}-I(t) d t=-\int_{t_{1}}^{T} \lambda_{0}\left[\frac{1-\delta T}{1-\beta_{1}}\left(t_{1}^{1-\beta_{1}}-t^{1-\beta_{1}}\right)+\frac{\delta}{2-\beta_{1}}\left(t_{1}^{2-\beta_{1}}-t^{2-\beta_{1}}\right)\right] d t
$$




$$
\begin{gathered}
\lambda_{0}\left[\frac{1-\delta T}{\left(1-\beta_{1}\right)\left(2-\beta_{1}\right)}\left(T^{2-\beta_{1}}-t_{1}^{2-\beta_{1}}\right)+\frac{\delta}{\left(2-\beta_{1}\right)\left(3-\beta_{1}\right)}\left(T^{3-\beta_{1}}-t_{1}^{3-\beta_{1}}\right)\right. \\
\left.-\left(\frac{1-\delta T}{1-\beta_{1}} t_{1}^{1-\beta_{1}}+\frac{\delta}{2-\beta_{1}} t_{1}^{2-\beta_{1}}\right)\left(T-t_{1}\right)\right]
\end{gathered}
$$

The amount of lost sales during the period $\left[t_{1}, \mathrm{~T}\right]$ is given by

$$
\begin{aligned}
L_{T} & =\int_{t_{1}}^{T} R(t)\left\{1-\frac{1}{1+\delta(T-t)}\right\} d t=\int_{t_{1}}^{T} \lambda_{0} t^{-\beta_{1}}\left\{1-\frac{1}{1+\delta(T-t)}\right\} d t \\
& =\lambda_{0} \delta\left[\frac{T}{1-\beta_{1}}\left(T^{1-\beta_{1}}-t_{1}^{1-\beta_{1}}\right)-\frac{1}{2-\beta_{1}}\left(T^{2-\beta_{1}}-t_{1}^{2-\beta_{1}}\right)\right]
\end{aligned}
$$

\subsection{Cost Components}

The total cost over the period $[0, \mathrm{~T}]$ consists of the following cost components:

(1) Ordering cost (OC)over the period $[0, \mathrm{~T}]=A_{0}$ (fixed)

(2) Purchasing cost (PC) over the period $[0, \mathrm{~T}]=p_{c} \mathrm{I}(0)=p_{c} \mathrm{Q}$

$$
=p_{c}\left\{\lambda_{0}\left(\frac{1}{1-\beta_{1}} t_{1}^{1-\beta_{1}}+\frac{\theta}{2-\beta_{1}} t_{1}^{2-\beta_{1}}-\frac{\alpha}{1-\beta_{1}+\beta} t_{1}^{1-\beta_{1}+\beta}\right)\right\}
$$

(3) Holding cost for carrying inventory (HC)over the period $[0, \mathrm{~T}]=h_{c} I_{T}$

$$
=h_{c} \lambda_{0}\left\{\frac{1}{2-\beta_{1}} t_{1}^{2-\beta_{1}}+\frac{\theta}{2\left(3-\beta_{1}\right)} t_{1}^{3-\beta_{1}}-\frac{\alpha \beta}{(1+\beta)\left(2-\beta_{1}+\beta\right)} t_{1}^{2-\beta_{1}+\beta}\right\}
$$

(4) Cost due to deterioration (CD) over the period $[0, T]=d_{c} D_{T}$

$$
=d_{c} \theta \lambda_{0}\left\{\frac{1}{2-\beta_{1}} t_{1}^{2-\beta_{1}}+\frac{\theta}{2\left(3-\beta_{1}\right)} t_{1}^{3-\beta_{1}}-\frac{\alpha \beta}{(1+\beta)\left(2-\beta_{1}+\beta\right)} t_{1}^{2-\beta_{1}+\beta}\right\}
$$

(5) The amelioration $\operatorname{cost}(\mathbf{A M C})$ over the period $[0, \mathrm{~T}]=a_{c} A_{T}$

$$
=\frac{a_{c} \lambda_{0} \alpha}{1-\beta_{1}+\beta} t_{1}^{1-\beta_{1}+\beta}
$$

(6) Cost due to shortage (CS) over the period $[0, \mathrm{~T}]=c_{s} S_{T}$

$$
=c_{s} \lambda_{0}\left[\frac{1-\delta T}{\left(1-\beta_{1}\right)\left(2-\beta_{1}\right)}\left(T^{2-\beta_{1}}-t_{1}^{2-\beta_{1}}\right)+\frac{\delta}{\left(2-\beta_{1}\right)\left(3-\beta_{1}\right)}\left(T^{3-\beta_{1}}-t_{1}^{3-\beta_{1}}\right)-\left(\frac{1-\delta T}{1-\beta_{1}} t_{1}^{1-\beta_{1}}+\frac{\delta}{2-\beta_{1}} t_{1}^{2-\beta_{1}}\right)\left(T-t_{1}\right)\right]
$$

(7) Opportunity cost due to lost sales (OPC) over the period $[0, T]=o_{c} L_{T}$

$$
=o_{c} \lambda_{0} \delta\left[\frac{T}{1-\beta_{1}}\left(T^{1-\beta_{1}}-t_{1}^{1-\beta_{1}}\right)-\frac{1}{2-\beta_{1}}\left(T^{2-\beta_{1}}-t_{1}^{2-\beta_{1}}\right)\right]
$$


The average total cost per unit time of the system during the cycle $[0, \mathrm{~T}]$ will be

$$
\begin{gathered}
\mathrm{TC}\left(t_{1}\right)=\frac{1}{T}[\mathbf{O C}+\mathbf{P C}+\mathbf{H C}+\mathbf{C D}+\mathbf{A M C}+\mathbf{C S}+\mathbf{O P C}] \\
=\frac{1}{T}\left[A_{0}+p_{c}\left\{\lambda_{0}\left(\frac{1}{1-\beta_{1}} t_{1}^{1-\beta_{1}}+\frac{\theta}{2-\beta_{1}} t_{1}^{2-\beta_{1}}-\frac{\alpha}{1-\beta_{1}+\beta} t_{1}^{1-\beta_{1}+\beta}\right)\right\}+\right. \\
\left(h_{c}+\theta d_{c}\right) \lambda_{0}\left\{\frac{1}{2-\beta_{1}} t_{1}^{2-\beta_{1}}+\frac{\theta}{2\left(3-\beta_{1}\right)} t_{1}^{3-\beta_{1}}-\frac{\alpha \beta}{(1+\beta)\left(2-\beta_{1}+\beta\right)} t_{1}^{2-\beta_{1}+\beta}\right\} \\
+\frac{a_{c} \lambda_{0} \alpha}{1-\beta_{1}+\beta} t_{1}^{1-\beta_{1}+\beta}+c_{s} \lambda_{0}\left[\frac{1-\delta T}{\left(1-\beta_{1}\right)\left(2-\beta_{1}\right)}\left(T^{2-\beta_{1}}-t_{1}^{2-\beta_{1}}\right)+\frac{\delta}{\left(2-\beta_{1}\right)\left(3-\beta_{1}\right)}\left(T^{3-\beta_{1}}-t_{1}^{3-\beta_{1}}\right)-\right. \\
\left.\left.\left(\frac{1-\delta T}{1-\beta_{1}} t_{1}^{1-\beta_{1}}+\frac{\delta}{2-\beta_{1}} t_{1}^{2-\beta_{1}}\right)\left(T-t_{1}\right)\right]+o_{c} \lambda_{0} \delta\left[\frac{T}{1-\beta_{1}}\left(T^{1-\beta_{1}}-t_{1}^{1-\beta_{1}}\right)-\frac{1}{2-\beta_{1}}\left(T^{2-\beta_{1}}-t_{1}^{2-\beta_{1}}\right)\right]\right]
\end{gathered}
$$

For minimum, the necessary condition is $\frac{d T C\left(t_{1}\right)}{d t_{1}}=0$

This gives $p_{c}\left(1+\theta t_{1}-\alpha t_{1}^{\beta}\right)+\left(h_{c}+\theta d_{c}\right)\left(t_{1}+\frac{\theta}{2} t_{1}^{2}-\frac{\alpha \beta}{1+\beta} t_{1}^{1+\beta}\right)+a_{c} \alpha t_{1}^{\beta}+$

$$
c_{s}\left(T-t_{1}\right)\left(1+\delta t_{1}-\delta T\right)-o_{c} \delta\left(T-t_{1}\right)=0
$$

For minimum the sufficient condition $\frac{\mathrm{d}^{2} \mathrm{TC}\left(\mathrm{t}_{1}\right)}{\mathrm{dt}_{1}{ }^{2}}>0$ would be satisfied.

Let $t_{1}=t_{1}^{*}$ be the optimum value of $t_{1}$.

The optimal values $Q^{*}$ of $\mathrm{Q}$ and $T C^{*}$ of TC are obtained by putting the value $t_{1}=t_{1}^{*}$ from the expressions (6) and (12).

\section{Some Particular Cases}

a) Absence of deterioration

If the deterioration of items is switched off i.e. $\theta=0$, then the expressions (6) and (12) of on-hand inventory (Q) and average total cost per unit time $\left(\mathrm{TC}\left(t_{1}\right)\right)$ during the period $[0, \mathrm{~T}]$ become

$$
\mathrm{Q}=\lambda_{0}\left(\frac{1}{1-\beta_{1}} t_{1}^{1-\beta_{1}}-\frac{\alpha}{1-\beta_{1}+\beta} t_{1}^{1-\beta_{1}+\beta}\right)
$$


And TC $\left(t_{1}\right)=\frac{1}{T}\left[A_{0}+p_{c}\left\{\lambda_{0}\left(\frac{1}{1-\beta_{1}} t_{1}^{1-\beta_{1}}-\frac{\alpha}{1-\beta_{1}+\beta} t_{1}^{1-\beta_{1}+\beta}\right)\right\}+\right.$
$h_{c} \lambda_{0}\left\{\frac{1}{2-\beta_{1}} t_{1}^{2-\beta_{1}}-\frac{\alpha \beta}{(1+\beta)\left(2-\beta_{1}+\beta\right)} t_{1}^{2-\beta_{1}+\beta}\right\}+\frac{a_{c} \lambda_{0} \alpha}{1-\beta_{1}+\beta} t_{1}^{1-\beta_{1}+\beta}+$
$c_{s} \lambda_{0}\left[\frac{1-\delta T}{\left(1-\beta_{1}\right)\left(2-\beta_{1}\right)}\left(T^{2-\beta_{1}}-t_{1}^{2-\beta_{1}}\right)+\frac{\delta}{\left(2-\beta_{1}\right)\left(3-\beta_{1}\right)}\left(T^{3-\beta_{1}}-t_{1}^{3-\beta_{1}}\right)-\right.$
$\left.\left.\left(\frac{1-\delta T}{1-\beta_{1}} t_{1}^{1-\beta_{1}}+\frac{\delta}{2-\beta_{1}} t_{1}^{2-\beta_{1}}\right)\left(T-t_{1}\right)\right]+o_{c} \lambda_{0} \delta\left[\frac{T}{1-\beta_{1}}\left(T^{1-\beta_{1}}-t_{1}^{1-\beta_{1}}\right)-\frac{1}{2-\beta_{1}}\left(T^{2-\beta_{1}}-t_{1}^{2-\beta_{1}}\right)\right]\right]$

The equation (13) becomes

$p_{c}\left(1-\alpha t_{1}^{\beta}\right)+h_{c}\left(t_{1}-\frac{\alpha \beta}{1+\beta} t_{1}^{1+\beta}\right)+a_{c} \alpha t_{1}^{\beta}+c_{s}\left(T-t_{1}\right)\left(1+\delta t_{1}-\delta T\right)-o_{c} \delta\left(T-t_{1}\right)=0$

This gives the optimum value of $t_{1}$.

b) Absence of amelioration

If the amelioration environment is not considered i.e. $\alpha=0$, then the expressions (6) and (12) of on-hand inventory (Q) and average total cost per unit time (TC $\left.\left(t_{1}\right)\right)$ during the period $[0, \mathrm{~T}]$ become

$$
\begin{gathered}
\mathrm{Q}=\lambda_{0}\left(\frac{1}{1-\beta_{1}} t_{1}^{1-\beta_{1}}+\frac{\theta}{2-\beta_{1}} t_{1}^{2-\beta_{1}}\right) \\
\text { And TC }\left(t_{1}\right)=\frac{1}{T}\left[A_{0}+p_{c}\left\{\lambda_{0}\left(\frac{1}{1-\beta_{1}} t_{1}^{1-\beta_{1}}+\frac{\theta}{2-\beta_{1}} t_{1}^{2-\beta_{1}}\right)\right\}+\right. \\
\left(h_{c}+\theta d_{c}\right) \lambda_{0}\left\{\frac{1}{2-\beta_{1}} t_{1}^{2-\beta_{1}}+\frac{\theta}{2\left(3-\beta_{1}\right)} t_{1}^{3-\beta_{1}}\right\} \\
+c_{s} \lambda_{0}\left[\frac{1-\delta T}{\left(1-\beta_{1}\right)\left(2-\beta_{1}\right)}\left(T^{2-\beta_{1}}-t_{1}^{2-\beta_{1}}\right)+\frac{\delta}{\left(2-\beta_{1}\right)\left(3-\beta_{1}\right)}\left(T^{3-\beta_{1}}-t_{1}^{3-\beta_{1}}\right)-\right. \\
\left.\left.\left(\frac{1-\delta T}{1-\beta_{1}} t_{1}^{1-\beta_{1}}+\frac{\delta}{2-\beta_{1}} t_{1}^{2-\beta_{1}}\right)\left(T-t_{1}\right)\right]+o_{c} \lambda_{0} \delta\left[\frac{T}{1-\beta_{1}}\left(T^{1-\beta_{1}}-t_{1}^{1-\beta_{1}}\right)-\frac{1}{2-\beta_{1}}\left(T^{2-\beta_{1}}-t_{1}^{2-\beta_{1}}\right)\right]\right]
\end{gathered}
$$

The equation (13) becomes

$$
p_{c}\left(1+\theta t_{1}\right)+\left(h_{c}+\theta d_{c}\right)\left(t_{1}+\frac{\theta}{2} t_{1}^{2}\right)+c_{s}\left(T-t_{1}\right)\left(1+\delta t_{1}-\delta T\right)-o_{c} \delta\left(T-t_{1}\right)=0
$$

This gives the optimum value of $t_{1}$.

c) If the demand rate is constant then $\beta_{1}=0$ : 
The expressions (6) and (12) of on-hand inventory (Q) and average total cost per unit time $\left(\mathrm{TC}\left(t_{1}\right)\right)$ during the period $[0, \mathrm{~T}]$ become

$$
\begin{gathered}
\mathrm{Q}=\lambda_{0}\left(t_{1}+\frac{\theta}{2} t_{1}^{2}-\frac{\alpha}{1+\beta} t_{1}^{1+\beta}\right) \\
\text { And } \mathrm{TC}\left(t_{1}\right)=\frac{1}{T}\left[A_{0}+p_{c}\left\{\lambda_{0}\left(t_{1}+\frac{\theta}{2} t_{1}^{2}-\frac{\alpha}{1+\beta} t_{1}^{1+\beta}\right)\right\}+\right. \\
\left(h_{c}+\theta d_{c}\right) \lambda_{0}\left\{\frac{1}{2} t_{1}^{2}+\frac{\theta}{6} t_{1}^{3}-\frac{\alpha \beta}{(1+\beta)(2+\beta)} t_{1}^{2+\beta}\right\}+\frac{a_{c} \lambda_{0} \alpha}{1+\beta} t_{1}^{1+\beta}+ \\
\left.c_{s} \lambda_{0}\left[\frac{1-\delta T}{2}\left(T^{2}-t_{1}^{2}\right)+\frac{\delta}{6}\left(T^{3}-t_{1}^{3}\right)-\left\{(1-\delta T) t_{1}+\frac{\delta}{2} t_{1}^{2}\right\}\left(T-t_{1}\right)\right]+o_{c} \lambda_{0} \delta\left[T\left(T-t_{1}\right)-\frac{1}{2}\left(T^{2}-t_{1}^{2}\right)\right]\right]
\end{gathered}
$$

The equation (13) becomes

$$
\begin{aligned}
& p_{c}\left(1+\theta t_{1}-\alpha t_{1}^{\beta}\right)+\left(h_{c}+\theta d_{c}\right)\left(t_{1}+\frac{\theta}{2} t_{1}{ }^{2}-\frac{\alpha \beta}{1+\beta} t_{1}^{1+\beta}\right)+a_{c} \alpha t_{1}^{\beta}+ \\
& c_{s}\left(T-t_{1}\right)\left(1+\delta t_{1}-\delta T\right)-o_{c} \delta\left(T-t_{1}\right)=0
\end{aligned}
$$

This gives the optimum value of $t_{1}$.

\section{Numerical Example}

To illustrate the developed inventory model, let the values of parameters be as follows:

$A_{0}=\$ 500$ per order; $\lambda_{0}=20 ; \beta_{1}=0.5 ; \theta=0.01 ; \alpha=0.001 ; \beta=2 ; p_{c}=\$ 5$ per unit, $h_{c}=\$ 12$ per unit; $d_{c}=\$ 4$ per unit; $a_{c}=\$ 7$ per unit; $c_{s}=\$ 10$ per unit; $o_{c}=\$ 12$ per unit; $\delta=10 ; \mathrm{T}=1$ year

Solving the equation (13) with the help of computer using the above values of parameters, we find the following optimum outputs

$t_{1}^{*}=0.87$ year; $Q^{*}=37.47$ units and $T C^{*}=$ Rs. 839.17

It is checked that this solution satisfies the sufficient condition for optimality.

\section{Sensitivity Analysis and Discussion}

We now study the effects of changes in the system parameters $\lambda_{\mathrm{o}}, \beta_{1}, \theta, \alpha, \beta, p_{c}, h_{c}, d_{c}, a_{c}, c_{s}, o_{c}$ and $\delta$ on the optimal on-hand inventory quantity $\left(Q^{*}\right)$ and the optimal total cost $\left(T C^{*}\right)$ in the present inventory model. The sensitivity analysis is performed by changing each of the parameters by $-50 \%$, $20 \%$, $+20 \%$ and $+50 \%$, taking one parameter at a time and keeping remaining parameters unchanged. The results are furnished in Table 1. 
Table 1: Effect of changes in the parameters on the model

\begin{tabular}{|c|c|c|c|}
\hline \multirow[t]{2}{*}{ Changing parameter } & \multirow{2}{*}{$\begin{array}{c}\text { \% change in the system } \\
\text { parameter }\end{array}$} & \multicolumn{2}{|c|}{$\%$ change in } \\
\hline & & $Q^{*}$ & $T C^{*}$ \\
\hline \multirow{4}{*}{$\lambda_{0}$} & -50 & -50 & -20.20 \\
\hline & -20 & -20 & -08.08 \\
\hline & +20 & +20 & 08.08 \\
\hline & +50 & +50 & 20.20 \\
\hline \multirow{4}{*}{$\beta_{1}$} & -50 & -35.51 & -10.66 \\
\hline & -20 & -17.76 & -05.16 \\
\hline & +20 & 26.66 & 07.32 \\
\hline & +50 & 46.69 & 20.20 \\
\hline \multirow{4}{*}{$\theta$} & -50 & -0.12 & -0.08 \\
\hline & -20 & -0.05 & -0.03 \\
\hline & +20 & 0.05 & 0.03 \\
\hline & +50 & 0.12 & 0.08 \\
\hline \multirow{4}{*}{$\alpha$} & -50 & 0.009 & 0.001 \\
\hline & -20 & 0.003 & 0.004 \\
\hline & +20 & -0.003 & -0.004 \\
\hline & +50 & -0.007 & -0.001 \\
\hline \multirow{4}{*}{$\beta$} & -50 & -0.01 & 0.005 \\
\hline & -20 & -0.03 & 0.002 \\
\hline & +20 & 0.03 & -0.002 \\
\hline & +50 & 0.02 & -0.003 \\
\hline \multirow{4}{*}{$p_{c}$} & -50 & 0.99 & 0.07 \\
\hline & -20 & 0.39 & 0.02 \\
\hline & +20 & -0.39 & -0.01 \\
\hline & +50 & -0.98 & -0.06 \\
\hline \multirow{4}{*}{$h_{c}$} & -50 & 2.17 & -8.03 \\
\hline & -20 & 0.84 & -3.14 \\
\hline & +20 & -0.80 & 3.05 \\
\hline & +50 & $-1-1.95$ & 7.46 \\
\hline \multirow{4}{*}{$d_{c}$} & -50 & 0.007 & -0.03 \\
\hline & -20 & 0.003 & -0.01 \\
\hline & +20 & -0.003 & 0.01 \\
\hline & +50 & -0.007 & 0.03 \\
\hline \multirow{4}{*}{$a_{c}$} & -50 & -0.002 & -0.002 \\
\hline & -20 & -0.0004 & -0.001 \\
\hline & +20 & 0.0004 & 0.001 \\
\hline & $\begin{array}{r}+50 \\
\end{array}$ & 0.001 & 0.002 \\
\hline \multirow{4}{*}{$c_{s}$} & -50 & -0.07 & -0.02 \\
\hline & -20 & -0.03 & -0.007 \\
\hline & +20 & 0.03 & 0.007 \\
\hline & +50 & 0.06 & 0.02 \\
\hline \multirow{4}{*}{$o_{c}$} & -50 & -2.70 & -2.51 \\
\hline & -20 & -1.40 & -0.65 \\
\hline & +20 & 1.03 & 0.43 \\
\hline & +50 & 2.13 & 2.54 \\
\hline \multirow{4}{*}{$\delta$} & -50 & -2.25 & -2.23 \\
\hline & -20 & -1.62 & -0.60 \\
\hline & +20 & 1.11 & 0.41 \\
\hline & +50 & 2.24 & 2.25 \\
\hline
\end{tabular}


Analyzing the results of Table 1, the following observations may be made:

(i) $T C^{*}$ increases or decreases with the increase or decrease in the values of the system parameters $\lambda_{0}, \beta_{1}, \theta, h_{c}, d_{c}, a_{c}, c_{s}, o_{c}$ and $\delta$. On the other hand $T C^{*}$ increases or decreases with the decrease or increase in the values of the system parameters $\alpha, \beta$ and $p_{c}$. The results obtained show that $T C^{*}$ is highly sensitive towards changes of ordering $\operatorname{cost} \lambda_{0}$ and $\beta_{1}$, moderate sensitive towards the changes of $h_{c}, o_{c}$ and $\delta$. It is less sensitive towards the changes of $\theta, \alpha, \beta, p_{c}, d_{c}, a_{c}$ and $c_{s}$.

(ii) $Q^{*}$ increases or decreases with the increase or decrease in the values of the system parameters $\lambda_{0}, \beta_{1}, \theta, \beta, c_{s}, o_{c}$ and $\delta$. On the other hand $Q^{*}$ increases or decreases with the decrease or increase in the values of the system parameters $\alpha, p_{c}, h_{c}, d_{c}$ and $a_{c}$. The results obtained show that $Q^{*}$ is highly sensitive towards changes of $\lambda_{0}$ and $\beta_{1}$, moderate sensitive towards the changes of $h_{c}, o_{c}$ and $\delta$. It is less sensitive towards the changes of $\theta, \alpha, \beta, p_{c}, d_{c}, a_{c}$ and $c_{s}$.

From the above analysis, it is concluded that the demand parameters $\lambda_{0}$ and $\beta_{1}$ are highly sensitive for both optimal values of $\mathrm{Q}$ and TC. So these parameters play an important role to evaluate optimal values and any error in the estimation of $\lambda_{0}$ and $\beta_{1}$ results in significant errors in the optimal cost. Hence, proper adequate attention must be taken to estimate these two demand parameters. 


\section{References}

[1] Hwang, H.S. (1997). A study of an inventory model for items with Weibull ameliorating, Computers and Industrial Engineering, 33, pp.701-704.

[2] Hwang, H.S. (2004). A stochastic set-covering location model for both ameliorating and deteriorating items, Computers and Industrial Engineering, 46, pp.313-319.

[3] Mallick M., Mishra S., Mishra U. K. and Paikray S.K. (2018). Optimal inventory control for ameliorating, deteriorating items under time varying demand condition, Journal of Social Science Research, 3(1), pp. 166-173.

[4] Moon I., Giri B.C. and B. Ko (2005). Economic order quantity models for ameliorating/deteriorating items under inflation and time discounting, European Journal of Operational Research, 162( 3), pp. 773-785.

[5] Law S.-T. and Wee H.M. (2006). An integrated production-inventory model for ameliorating and deteriorating items taking account of time discounting, Mathematical and Computer Modelling, v 43(5-6), pp. 673-685.

[6] L.-Q. Ji. (2008). The influences of inflation and time-value of money on an EOQ model for both ameliorating and deteriorating items with partial backlogging, in Proceedings of the 2008 International Conference on Wireless Communications, Networking and Mobile Computing, WiCOM, Dalian, China.

[7] Valliathal, M. and Uthayakumar R. (2010). The production-inventory problem for ameliorating/deteriorating items with non-linear shortage cost under inflation and time discounting, Applied Mathematical Sciences, 4(5-8), pp. 289-304.

[8] Barik, S., Mishra, S., Paikray, S.K. and Misra, U.K. (2013). An Inventory Model for Weibull Ameliorating, Deteriorating Items under the Influence of Inflation, International Journal of Engineering Research and Applications, 3, pp. $1430-1436$.

[9] L. Tadj, A.M. Sarhan and A. EI-Gohary, (2008). Optimal control of an inventory system with ameliorating and deteriorating items, Applied Sciences, 10, pp. 243-255.

[10] S. Y. Chou, W. T. Chouhuang, J. S. J. Lin and P. Chu, (2008). An analytic solution approach for the economic order quantity model with Weibull ameliorating items, Math. Comput. Model, 48, pp.1868-1874.

[11] B. Mondal, A. K. Bhunia and M. Maiti, (2003). An inventory system of ameliorating items for price dependent demand rate, Comput. Indust. Eng., 45, pp.443-456.

[12] Donaldson W.A. (1977). Inventory replenishment policy for a linear trend in demand-an analytical solution. Operational Research Quarterly, 28, pp. 663670.

[13] Silver, E.A. (1979). A simple inventory replenishment decision rule for a linear trend in demand. Journal of Operational Research Society. 30, pp.71-75. 
[14] Goswami, A. and Chaudhri, K.S. (1991). An EOQ model for deteriorating items with a linear trend in demand. Journal of Operational Research Society. 42(12), pp.1105-1110.

[15] Buchanan J. T. (1980). Alternative solution methods for the inventory replenishment problem under increasing demand, J Opl Res. Soc., 31, pp.615620.

[16] Dutta T. K. And Pal A. K. (1992). A note on a replenishment policy for an inventory model with linear trend in demand and shortages, J Opl Res. Soc., 43, pp.993-1001.

[17] Biswaranjan Mandal, (2020). An Optimal Inventory Policy for Deteriorating Items with Additive Exponential Life Time having Linear Trended Demand and Shortages, International Journal of Engineering and Technical Research, 10(9), pp. 12-15.

[18] Goyal S.K., Morin D. and Nebebe F. (1992). The finite horizon trended inventory replenishment problem with shortages, J. Opl. Res. Soc., 43, pp. 1173-1178.

[19] Benkherouf L. (1995). On an inventory model with deteriorating items and decreasing time- varying demand and shortages, Eur. J. Opl. Res., 86, pp.293299.

[20] Wee H.M. (1995). A deterministic lot-size inventory model for deteriorating items with shortages and declining market, Comp. Oper. Res., 22, pp.345356.

[21] Biswaranjan Mandal (2020). An inventory management for deteriorating and ameliorating items with a linear trended in demand and partial backlogging, Global Journal of Pure and Applied Mathematics, 16(6), pp. 759-770. 